\title{
Can public sector community health workers deliver a nurturing care intervention in South Africa? The Amagugu Asakhula feasibility study
}

\author{
Sonja Klingberg ${ }^{1,2^{*}}$ (D), Esther M. F. van Sluijs ${ }^{1,2}$, Stephanie T. Jong ${ }^{3}$ and Catherine E. Draper ${ }^{2,4}$
}

\begin{abstract}
Background: Nurturing care interventions have the potential to promote health and development in early childhood. Amagugu Asakhula was designed to promote developmentally important dietary and movement behaviours among children of preschool age (3-5 years) in South Africa. An initial formative study in Cape Town found the intervention to be feasible and acceptable when delivered by community health workers (CHWs) linked to a community-based organisation. This study evaluated the delivery of the Amagugu Asakhula intervention by CHWs linked to a public sector primary health care facility in Soweto, as this mode of delivery could have more potential for sustainability and scalability.
\end{abstract}

Methods: A qualitative design was utilised to assess feasibility, acceptability, adoption, appropriateness, implementation, fidelity and context. CHWs $(n=14)$ delivered the intervention to caregivers $(n=23)$ of preschoolage children in Soweto over 6 weeks. Following the completion of the intervention, focus group discussions were held with CHWs and caregivers. Further data were obtained through observations, study records and key informant interviews $(n=5)$. Data were analysed using deductive thematic analysis guided by a process evaluation framework. Results: The delivery of the Amagugu Asakhula intervention through CHWs linked to a primary health care facility in Soweto was not found to be feasible due to contextual challenges such as late payment of salaries influencing CHW performance and willingness to deliver the intervention. CHWs expressed dissatisfaction with their general working conditions and were thus reluctant to take on new tasks. Despite barriers to successful delivery, the intervention was well received by both CHWs and caregivers and was considered a good fit with the CHWs' scope of work.

(Continued on next page)

\footnotetext{
* Correspondence: sonja.klingberg@wits.ac.za

${ }^{1}$ MRC Epidemiology Unit, UKCRC Centre for Diet and Activity Research (CEDA

R), University of Cambridge, Cambridge, UK

${ }^{2}$ SAMRC/Wits Developmental Pathways for Health Research Unit (DPHRU),

School of Clinical Medicine, Faculty of Health Sciences, University of the

Witwatersrand, Johannesburg, South Africa

Full list of author information is available at the end of the article
}

(c) The Author(s). 2021 Open Access This article is licensed under a Creative Commons Attribution 4.0 International License, which permits use, sharing, adaptation, distribution and reproduction in any medium or format, as long as you give appropriate credit to the original author(s) and the source, provide a link to the Creative Commons licence, and indicate if changes were made. The images or other third party material in this article are included in the article's Creative Commons licence, unless indicated otherwise in a credit line to the material. If material is not included in the article's Creative Commons licence and your intended use is not permitted by statutory regulation or exceeds the permitted use, you will need to obtain permission directly from the copyright holder. To view a copy of this licence, visit http://creativecommons.org/licenses/by/4.0/ The Creative Commons Public Domain Dedication waiver (http://creativecommons.org/publicdomain/zero/1.0/) applies to the data made available in this article, unless otherwise stated in a credit line to the data. 
(Continued from previous page)

Conclusions: Based on these findings, delivery of the Amagugu Asakhula intervention is not recommended through public sector CHWs in South Africa. This feasibility study informs the optimisation of implementation and supports further testing of the intervention's effectiveness when delivered by CHWs linked to community-based organisations. The present study further demonstrates how implementation challenges can be identified through qualitative feasibility studies and subsequently addressed prior to large-scale trials, avoiding the wasting of research and resources.

Keywords: Nurturing care, Community health workers, South Africa, Qualitative research, Process evaluation, Feasibility study

\section{Key messages regarding feasibility}

- Integrating nurturing care into existing systems has been recommended in low- and middle-income countries with scarce resources for scaling up interventions, but there were uncertainties regarding the feasibility of public sector community health workers delivering such interventions in South Africa.

- Our qualitative findings illustrate how contextual challenges may hinder such integration, as intervention delivery by public sector community health workers was not found to be feasible.

- Delivery by community health workers linked to community-based organisations instead of primary health care facilities is recommended for future trials of the Amagugu Asakhula intervention.

\section{Background}

Nurturing care has become increasingly recognised as a key framework for early childhood development [1]. Defined as "a stable environment that is sensitive to children's health and nutritional needs, with protection from threats, opportunities for early learning, and interactions that are responsive, emotionally supportive, and developmentally stimulating" [1] (p. 91), nurturing care largely aims to ensure that children grow and develop to reach their full potential. Nurturing care interventions in early childhood are a promising avenue for addressing a multitude of child health and developmental challenges, such as different forms of malnutrition and developmental delay, in low- and middle-income countries (LMICs) [2, 3].

The importance of promoting early childhood development is well established and widely recognised, but the implementation and scale-up of interventions that can achieve such outcomes in LMIC settings need to be enhanced $[4,5]$. In particular, it is important to understand dimensions such as delivery, feasibility, context, and integration of interventions into existing systems [4, 6]. These dimensions can be examined through process evaluations and formative research, which are recommended components of intervention development and testing [7, 8]. Formative studies, such as pilot and feasibility studies, provide research insights at a small scale to help to determine whether an intervention is appropriate for further testing [9], enable optimisation of interventions prior to larger evaluations or wider implementation, and support decisions regarding definitive effectiveness trials [10-12]. The embedment of process evaluations and formative research, particularly in implementation research in LMIC settings, has been called for in order to maximise learning about interventions and avoid wasting scarce resources [5, 13, 14].

Following the success of a South African paediatric HIV disclosure intervention called Amagugu [15-17], the intervention was adapted into an early childhood health and development intervention called Amagugu Asakhula ("treasures that are still growing"). While certain key elements of the original Amagugu intervention were retained (caregiver support, strengthening the caregiver/child relationship, counselling approach, and home-based delivery), the original HIV messages were replaced with messages focussing on children's cognitive development, physical activity, screen time, diet, and sleep. This adapted intervention aligns with the nurturing care framework [1], and its theory of change is described in detail elsewhere [3]. Amagugu Asakhula is delivered by Community Health Workers (CHWs) to individual caregivers of preschool-age children through weekly sessions over a six-week period. It intends to promote nurturing interactions and developmentally important health behaviours.

An initial pilot study of the Amagugu Asakhula intervention in a low-income urban setting in Cape Town, South Africa, found it to be both feasible and acceptable for caregivers of preschool children in a low-income urban setting, when delivered by CHWs linked to a community-based organisation [3]. Based on this initial formative work, the Amagugu Asakhula intervention was deemed appropriate for further testing. The present study aimed to evaluate an alternative delivery mode through CHWs linked to a public primary health care 
(PHC) facility in Soweto, a predominantly low-income, urban setting in South Africa. Through making use of existing public sector structures, this delivery mode could have considerable potential for future implementation, including opportunities for scale-up, costeffectiveness and sustainability of the intervention, if Amagugu Asakhula is found to be effective.

Community-based PHC settings in LMICs are a potential entry point for nurturing care interventions, and the integration of new interventions with such existing platforms is recommended [1]. Public PHC in South Africa is organised around clinics and community health centres that are free at the point of use [18]. Through CHWs, PHC facilities can also carry out health promotion activities and community outreach and link with other public providers such as social services. Public sector CHWs are part of a ward-based outreach team (WBOT) system, which means that they are deployed in their local communities and attached to a public PHC facility [19]. $\mathrm{CHWs}$ are each responsible for a specified number of households in their community, and their tasks include, for instance, promoting antenatal care attendance, the immunisation of children, and treatment adherence in the case of chronic illnesses. CHWs are managed by team leaders who are trained nurses $[20,21]$ and receive formal training before being deployed in the communities. While some formalised processes are in place, there are notable gaps between policy and implementation in terms of the WBOT system and PHC more generally $[19,22]$. For example, linkages and referrals between different institutions and services are suboptimal in many cases, and there has been an insufficient focus on prevention and health promotion [22].
The primary aim of the current study was to evaluate the feasibility and acceptability of the delivery of Amagugu Asakhula by CHWs linked to a public PHC facility in Soweto, a predominantly low-income, urban setting in South Africa. A further aim was to generate contextspecific insights about implementation to support the optimisation of the intervention and its delivery in a new setting.

\section{Methods}

\section{Study design and setting}

This feasibility study examined feasibility and acceptability, as well as adoption, appropriateness, implementation, fidelity, and context $[12,15,16]$. Data collection methods included semi-structured key informant interviews, focus group discussions (FGDs) with CHWs and intervention participants, qualitative observations, sociodemographic background questionnaires, and tracking of recruitment, training, and implementation records. This study is reported in accordance with the TIDIeR and SRQR reporting guidelines [23, 24], as well as the consolidated advice for reporting early childhood development implementation research (CARE) guidelines [25]. The study process is illustrated in Fig. 1.

A specific PHC facility in Soweto was selected as the site for this study due to existing contacts with the facility and the site's openness to engage with research. Due to the sensitive nature of some findings and to preserve confidentiality, the facility in question and its location in Soweto will not be identified. Soweto is a densely populated urban township in Gauteng Province, South Africa. Due to the legacy of colonial and apartheid urban planning and policies of racial segregation, Soweto's population is predominantly Black African. Some socioeconomic diversity exists, most notably between

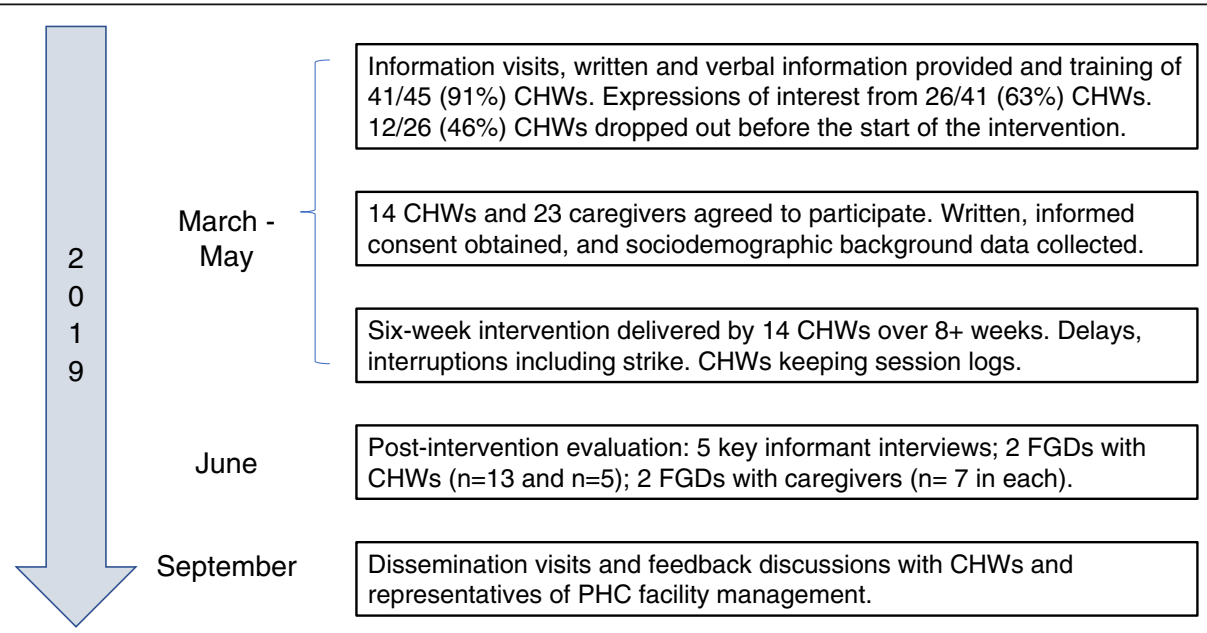

Fig. 1 Flowchart of the Amagugu Asakhula feasibility study 
very deprived areas considered informal settlements, and more formal middle-class neighbourhoods. The study setting was a predominantly low-income area with formal housing, characterised by permanent structures such as brick and cement houses, as opposed to more temporary structures such as shacks made of corrugated iron.

While the design of this feasibility study in Soweto is similar to that of the original pilot study in Cape Town [3], there are key differences in intervention delivery that warrant investigation. In Soweto CHWs were linked to a public PHC facility, while in Cape Town CHWs were linked to a community-based organisation. In Soweto, CHWs delivered the intervention individually, while in Cape Town CHWs worked in pairs due to different safety concerns [3]. In addition, CHWs in Soweto only delivered the intervention to one or two caregivers, while each pair of CHWs in Cape Town was required to deliver the intervention to ten caregivers. CHWs in Cape Town had a lower existing workload than CHWs in Soweto linked to a public PHC facility.

Ethical approval was obtained from the University of the Witwatersrand's Medical Human Research Ethics Committee (reference number M181063). All participating CHWs and caregivers gave informed written consent to participation and to being audio-recorded if relevant. Permission to carry out the study through a public PHC facility was also obtained from the Johannesburg Health District Research Committee (DRC Ref. 2019-01-013). After formal permission to conduct research at the facility had been obtained, SK provided information about the intervention and study to all CHWs, their managers and team leaders via information sheets. All CHWs and team leaders linked to the facility were invited to take part in the Amagugu Asakhula training sessions that prepared CHWs to deliver the intervention to families with preschool-age children.

\section{Training and recruitment}

CHWs attended two 4-h training sessions delivered by SK who had attended the training of CHWs in Cape Town and been trained by one of the intervention developers to deliver CHW training. The training was delivered in a seminar format with considerable practical examples and ample time for questions and discussions. The training sessions introduced the research aspects of the study, covered background information about child nutrition, health, and development, as well as the approach, content, and materials of the Amagugu Asakhula intervention. Topics were also detailed in an instruction manual that was distributed to each $\mathrm{CHW}$. Considerable focus was given to health-related behaviours according to South African guidelines on movement and dietary behaviours relevant for this age group [26-29] and how these relate to child nutrition, obesity prevention, and children learning and developing well. Expectations of participating CHWs, information, and materials were described in detail, both verbally and in the manual, along with the activities for CHWs to deliver to caregivers in each of the six sessions. Some individual tailoring was encouraged to communicate with caregivers in a relevant and respectful way. For example, when covering healthy dietary behaviours, CHWs were asked to align the discussions with each family's perceived circumstances and level of food security. Refreshments, including lunch, were provided to CHWs and team leaders attending the training sessions. CHWs who attended the training received certificates of attendance.

CHWs who attended training were asked to register their interest and intention to deliver the intervention. We employed a pragmatic approach to enable the budgeting of intervention materials for packs that would be purchased and distributed to CHWs. At the end of the second training session, information sheets, consent forms, and sociodemographic questionnaires were provided to CHWs in order for them to recruit caregivers of preschool-age children into the study. CHWs had 1 week to make contact with and recruit caregivers prior to delivering the intervention. All participating caregivers, and all CHWs who completed the intervention, were given ZAR200 ( US\$ 11) supermarket vouchers as appreciation for their time and input to the research.

\section{Study procedures and data collection}

The entire study was carried out between March and September 2019. The process is described in Fig. 1. To complete the intervention, $\mathrm{CHWs}$ were required to attend at least one training session and deliver six sessions over 6 weeks to the caregivers they recruited. As this was a pragmatic evaluation, participating $\mathrm{CHWs}$ were responsible for recruiting caregivers, obtaining informed written consent for the caregivers' participation, and background sociodemographic characteristics of participating caregivers (see Additional files). They were also asked to keep records of home visits and write brief notes after each intervention session using printed forms with specific questions (see Additional files).

Three weeks after the 6-week intervention had been completed, SK conducted five key informant interviews with individuals who had insights into the organisational structures and systemic dimensions of the WBOTs. The informants held positions of responsibility within the daily operations of the PHC facility. To preserve confidentiality of the interviews and respect the anonymity of the five individuals, these participants are not described in detail. 
In the same time period following the intervention, four FGDs were conducted: two with participating caregivers ( $n=7$ in each group), one FGD with CHWs who delivered the intervention $(n=13)$, and one FGD with CHWs who dropped out of delivering the intervention $(n=5)$. The FGDs were moderated by SK, with notetaking assistance from graduate students. All but one CHW delivering the intervention took part in the FGD. CHWs preferred to be in the same FGD, rather than divided into smaller groups. Refusals to participate in FGDs were due to other commitments (CHW, $n=1$ ), not wanting to be audio-recorded (caregivers, $n=2$ ), or not specified (caregivers, $n=7$ ).

All interviews and FGDs were conducted in English by SK (see Additional files for instruments used) and audiorecorded (Philips DVT4010 VoiceTracer). They were transcribed verbatim either by SK $(n=2$ FGDs with CHWs) or by a professional transcriber ( $n=2$ FGDs with caregivers and $n=5$ interviews). All professionally generated transcripts were checked by SK against original recordings. Field notes of observations were kept by SK during all visits to the PHC facility. Records were kept of training and meeting attendance, recruitment and retention of CHWs and caregivers, and all correspondence between SK and participating individuals.

In September 2019, study findings were shared by SK and CED with participating CHWs and other stakeholders. Feedback regarding the study was discussed over 2 days.

\section{Evaluation framework and data analysis}

Data analysis software MAXQDA (Release 12.2.0) was used to facilitate qualitative data analysis and management. Based on existing literature [7, 30], Table 1 outlines the applied process evaluation framework developed for evaluating the delivery the Amagugu Asakhula intervention.

Analysis followed the methods developed by Braun and Clarke for thematic analysis, but the analytic process comprised what can be called 'codebook' thematic analysis rather than the reflexive approach to thematic analysis [31-33]. Themes were developed a priori using a deductive (theoretical) approach, based on the process evaluation framework. Accordingly, the pre-developed themes were used as a starting point for the coding of both interview and FGD transcripts. Themes were divided into codes such as 'in support of feasibility' and 'against feasibility', and the proximity of the different participant types to the intervention informed the weight that was given to their views in forming conclusions. Coding was conducted by SK, and the results of the thematic analysis were discussed and refined by the full team of authors, utilising a 'critical friends' approach for enhancing rigour in the analysis [34]. Field observations were used to support interpretation and analysis.

\section{Results}

Table 2 provides an overview of the results depicted as illustrative quotations and organised according to the process evaluation framework. The findings describe how different aspects of the Amagugu Asakhula intervention and its delivery were received by both participating caregivers and the CHWs delivering it. Most importantly, it details barriers and facilitators to the specific intervention delivery approach, namely through public sector CHWs.

\section{Adoption}

In total, 91\% (41 out of 45) of CHWs attended at least one of the two training sessions; 18 attended both

Table 1 Process evaluation framework

\begin{tabular}{|c|c|c|}
\hline Concept & Questions addressed & Data sources \\
\hline Adoption & $\begin{array}{l}\text { To what extent were the training (CHWs) and intervention } \\
\text { (CHWs and caregivers) adopted by targeted groups? }\end{array}$ & $\begin{array}{l}\text { Recruitment and retention records, observations, separate FGDs } \\
\text { with CHWs, and caregivers after the intervention }\end{array}$ \\
\hline Acceptability & $\begin{array}{l}\text { To what extent was the intervention delivery agreeable and } \\
\text { satisfying, and perceived as acceptable by caregivers and CHWs? }\end{array}$ & Separate FGDs with CHWs and caregivers after the intervention \\
\hline Appropriateness & $\begin{array}{l}\text { How well does the intervention correspond with caregivers' } \\
\text { situations and needs? Are CHWs well suited for delivering this } \\
\text { intervention? }\end{array}$ & $\begin{array}{l}\text { Separate FGDs with CHWs and caregivers after intervention, } \\
\text { observations during training and intervention, feedback from } \\
\text { CHWs about training, and key informant interviews }\end{array}$ \\
\hline $\begin{array}{l}\text { Implementation } \\
\text { and fidelity }\end{array}$ & $\begin{array}{l}\text { To what extent was the intervention implemented as intended } \\
\text { in the design, and consistently with the underlying theory and } \\
\text { philosophy? }\end{array}$ & $\begin{array}{l}\text { Observations, key informant interviews, separate FGDs with } \\
\text { CHWs, and caregivers after intervention }\end{array}$ \\
\hline $\begin{array}{l}\text { Feasibility and } \\
\text { context }\end{array}$ & $\begin{array}{l}\text { How successfully can this intervention be carried out in this } \\
\text { setting? How successfully can the necessary evaluation } \\
\text { components of this intervention be carried out in this setting? } \\
\text { Are there contextual barriers or facilitators related to this } \\
\text { intervention and/or its evaluation? To what extent are they } \\
\text { modifiable, and do they necessitate further tailoring of the } \\
\text { intervention for a full trial? Were there any factors external to } \\
\text { the intervention that seemed to influence implementation? }\end{array}$ & $\begin{array}{l}\text { Recruitment and retention records, observations, key informant } \\
\text { interviews, separate FGDs with CHWs, and caregivers after } \\
\text { intervention }\end{array}$ \\
\hline
\end{tabular}




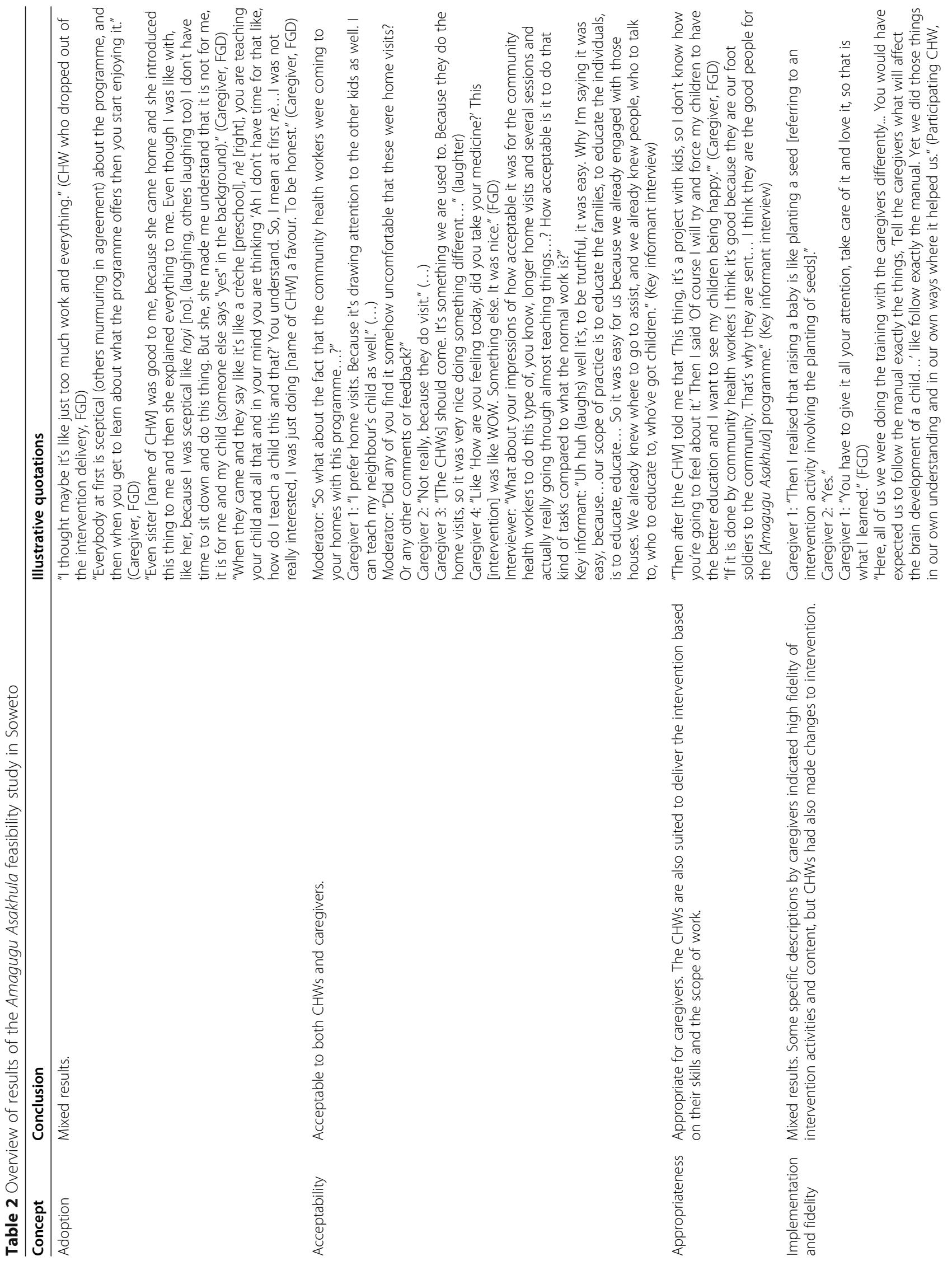




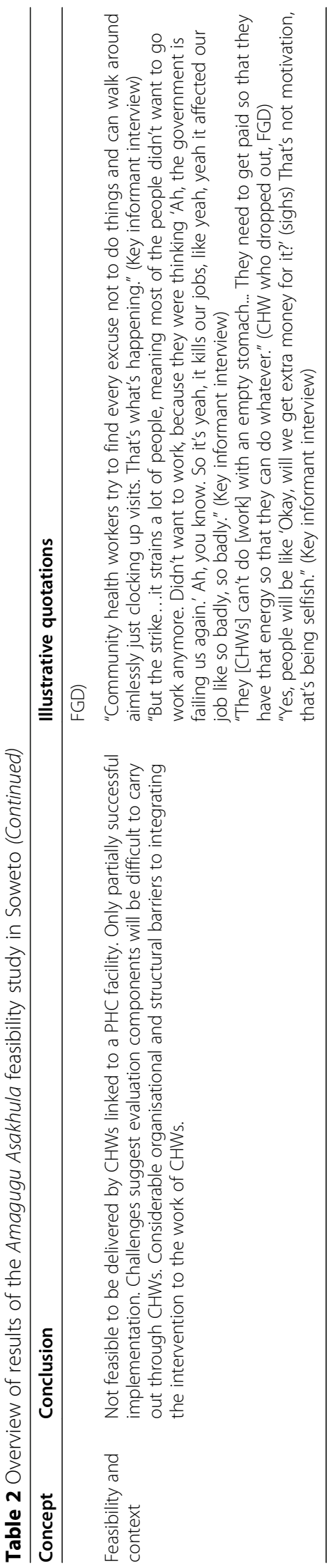


sessions. Subsequently, twenty-six CHWs expressed interest at this initial stage, agreeing to recruit 1-2 caregivers to deliver the intervention to over the subsequent 6 weeks. However, only 14 CHWs actually delivered the intervention, and 23 caregivers participated in the study.

Initial scepticism towards the intervention was reported by both CHWs and caregivers, which impacted upon their desire to participate in the intervention. Among CHWs, initially, the intervention was described as "extra work" or "too much work". Among caregivers, reasons for their initial scepticism included concerns about the required time commitment, perceptions that the intervention was "boring", the expectation of having to teach children things, learning how to raise children despite already having experience of raising children, and fears about the intervention putting children at risk of strangers. CHWs were able to mitigate these reservations in two ways: (1) using the existing relationships and trust built between CHWs and caregivers to reassure them about the study (Table 2) and (2) by providing more information. Children's interest in the intervention activities prompted caregivers to overcome their initial concerns, and they demonstrated more enthusiasm about Amagugu Asakhula.

It was also reported in both interviews and FGDs that there were financial barriers to adoption of the intervention. Key informants suggested that both CHWs and caregivers were likely to want incentives or compensation for participating in the intervention. Inability of the study to provide additional pay, on top of the challenges to CHWs receiving their regular salaries on time (unrelated to the study), reportedly played a considerable part in the lack of adoption from CHWs. CHWs who attended the training were informed that participation would not increase their salaries. As such, the reported delays in receiving their regular salaries may have been a more significant reason for the high number of CHWs who did not deliver the intervention after completing a training session $(n=12)$.

\section{Acceptability}

Caregivers did not express any objections to the intervention being delivered by CHWs and were enthusiastic about the convenience of the sessions taking place in their homes. Many of the participating caregivers were unemployed and described having plenty of time for the home-based intervention. Some caregivers described instances where CHWs turned up unannounced, causing some inconvenience. However, these situations were usually resolved by agreeing on specific times for Amagugu Asakhula sessions.

While it was normal for CHWs to visit families in the community, one key informant expressed concerns about having CHWs visit specific homes on a frequent basis as a potential barrier to acceptability from the caregivers' perspective due to the potential stigma attached to such visits. However, this concern about was not expressed by any caregivers, some of whom described telling neighbours about Amagugu Asakhula, and doing intervention activities with other children in their neighbourhoods. In turn, caregivers suggested incorporating group sessions or activities to the intervention itself. Caregivers expressed that participating in the FGD was a positive experience as it provided them the opportunity to hear about other families' experiences.

\section{Appropriateness}

Both CHWs and key informants reported that the Amagugu Asakhula intervention was a good fit with the regular scope of CHWs' work, and that they were able to carry out home visits thanks to being known and trusted by community members. According to key informants and CHWs themselves, CHWs linked to a PHC facility should, at least in theory, be able to accommodate this type of intervention within their work both in terms of both its content and focus on preschool-age children, as well as the home-based mode of delivery.

Similarly, the intervention resonated with the caregivers' situations as they were able to carry out intervention activities with their children. Caregivers described how the intervention aligned with their aspirations to ensure their children could learn well and be happy (Appropriateness and acceptability, Table 2). Caregivers who could not afford to send their children to preschool were particularly keen to support their children's learning and development through the activities and nurturing interactions promoted through Amagugu Asakhula. Some caregivers reported being surprised by how much their young children had learned in a short amount of time, and this sense of achievement, combined with the children's enjoyment, was central to the appeal of the intervention.

\section{Implementation and fidelity}

The delivery of the 6-week intervention was interrupted after the first week due to $\mathrm{CHW}$ strikes taking place across South Africa. During the 2 weeks that strikes took place, no Amagugu Asakhula sessions were run. Many of the CHWs reported not working during this time. Due to the delays, it took at least 8 weeks for CHWs to complete the sessions with participating caregivers. Data collection for the post-intervention evaluation was carried out 3 weeks after intervention delivery was completed.

Study findings denote differences in the delivery of Amagugu Asakhula compared to the intended design of the intervention. Based on caregivers' detailed descriptions of activities, and reflections that they shared with 
each other in FGDs, there were indications that many activities and topics had been covered by CHWs according to the manual. However, there was also some evidence that recruitment and delivery did not go according to the intervention's intended procedures and criteria. For example, some caregivers who participated in FGDs did not have children in the targeted age range, despite the confirmation from sociodemographic questionnaires returned by CHWs. Moreover, during the FGDs, it emerged that some CHWs had not yet started the intervention with participants by the time they had been invited to post-intervention FGDs. Due to the discrepancies in session records, the CHWs' accounts of sessions completed have been excluded as evidence of fidelity.

In addition to these indications of low fidelity, CHWs also reported in FGDs that they had made changes to the intervention. These changes included asking caregivers to study the intervention manual on their own, covering some sessions via WhatsApp messages or phone calls rather than in person, and skipping or combining some sessions. Some of these changes had been made in response to specific challenges, such as difficulties scheduling the weekly sessions. CHWs reported that the lack of close monitoring of the CHWs' activity enabled them to do things in their preferred way, as opposed to how the intervention had been intended.

CHWs discussed how it was possible for them to personalise the content, and communicate intervention messages in a way that they found helpful, as opposed to exactly how they were described in the intervention manual (Table 2). However, despite it being covered in the training provided, CHWs described that it was unclear which aspects of the intervention were flexible (e.g. how the content is phrased) and which aspects were not (e.g. inclusion criteria, and the number, order, delivery method, and focus of sessions with participating families).

\section{Feasibility and context}

As mentioned, both CHWs and key informants considered the intervention a good fit with the regular work and roles of CHWs linked to a public PHC facility in Soweto, at least in theory. Some key informants affirmed that the Amagugu Asakhula intervention could be scaled up and integrated into the WBOT system if found to be effective in the future. However, the CHWs' reluctance to have new or additional work incorporated into their existing roles presented a considerable barrier to feasibly implementing the Amagugu Asakhula intervention. Similarly, key informants who openly acknowledged this barrier were sceptical of how implementation could be done in practice. Thus, there were some contradictions both within and between the key informants' and CHWs' accounts.

Two clashing narratives about the challenges were expressed by CHWs and key informants respectively. CHWs emphasised that they had too much to do, while key informants indicated that the CHWs were not doing enough in their role. CHW's view of being overworked for their current salaries was closely linked to the strikes and labour union action taking place across the country at the time of the intervention. CHWs were unhappy with their precarious status; some reported that their contracts defined them as interns or trainees, rather than employees, and many were disgruntled with either their salaries, or not being paid on time. According to CHWs, introducing any additional or new work to their role would require an addition in salary, and strengthening their status as public sector health workers. The contrasting view denotes that CHWs are not monitored well enough. Due to this lack of accountability, as well as a perceived lack of motivation, commitment or work ethic, CHWs were thought of as doing less work than could reasonably be expected of them for their salary. As such, CHWs were perceived as being unwilling take on anything new.

While it is not possible to ascertain whether one of these views, a combination, or something else entirely, was the most accurate account of the situation, these organisational challenges and conflicts were described as considerable challenges to delivering Amagugu Asa$k h u l a$, as well as the WBOT system more broadly. It was recommended by both CHWs and key informants that the intervention could or should be delivered through or together with other actors, such as preschools and community-based organisations.

\section{Discussion}

This feasibility study has investigated the feasibility and acceptability of delivering the Amagugu Asakhula intervention through CHWs linked to a PHC facility in Soweto. The analysis indicates that the intervention and its delivery are both acceptable and, in theory, appropriate. However, due to contextual challenges, the delivery of the intervention is, in practice, not feasible via CHWs linked to a PHC facility. This is in line with other studies examining CHW systems in South Africa and other LMIC settings [20, 35-37], as CHWs have become a cadre of health workers to whom various new tasks are assigned, without necessarily much consultation or consideration of their capacity in terms of time, working conditions or skills.

The challenges of supervision and accountability around CHWs in South Africa have been previously recognised [5] and are not unique to South Africa. A study conducted in Pakistan similarly evaluated the 
integration of early childhood development interventions to an existing community health programme, and it highlights the importance of supportive $\mathrm{CHW}$ supervision, and the need for capacity building at the organisational level for improved implementation and scalability of the intervention [38]. The findings of the present feasibility study highlight the corresponding perspectives of the CHWs, who are hindered and demotivated by organisational issues, which understandably influence their performance. A systematic review of interventions to improve CHW performance in LMICs found potential for improvements through interventions such as tailored incentives, task reminders and an emphasis on career opportunities when recruiting CHWs [39]. However, the accounts of the Sowetan CHWs suggest that simply being paid on time and having more contractual stability might have a bearing on CHW' motivation and performance.

Some of the contextual challenges encountered in the present study provide an opportunity to optimise the intervention by, for example, modifying it to better accommodate delays and disruptions in the future. In particular, the strikes provided insight into the overall functioning of the WBOT system, and the PHC facility's operations more generally, which paints a more complex and volatile picture. Views expressed by both key informants and CHWs in FGDs pointed to issues with supervision, accountability, resistance to change, and practical challenges of balancing work in the community with demands for being present in the facility. Prior research in South Africa has also identified the lack of supervision or monitoring of the $\mathrm{CHW}$ role as a challenge for the WBOT system [40].

The delivery challenges and hesitant adoption of the intervention by CHWs linked to a public PHC have implications for the implementation of Amagugu Asa$k h u l a$. To ensure scalability and sustainability, existing public health system structures are recommended avenues for delivering interventions [1, 4]. Conversely, findings from the current study indicate that it may be more realistic to consider other options for delivering the Amagugu Asakhula intervention. Instead of public sector institutions, other health system actors, such as CHWs linked to community-based organisations, could deliver the intervention without being constrained by some of the challenges of the WBOTs. As the pilot study in Cape Town demonstrated, CHWs whose time is dedicated to the intervention, and compensated for, could deliver the intervention more successfully [3]. This route through communitybased organisations is not without its own challenges, such as higher costs and possibly limited potential for scalability and sustainability compared to that of the public sector. Implementation of $\mathrm{CHW}$ programmes through community-based organisations has also been criticised for lacking accountability [20], and it ignores the need to the strengthen community health systems through the public sector [41, 42].

The recommended process of integrating nurturing care interventions into existing delivery systems [4], such as the WBOT system in South Africa, may become realistic to pursue further in the future if rigorous evaluations demonstrate the effectiveness of the Amagugu Asakhula intervention. This study not only provides valuable insights into how the contextual challenges influence implementation, but it also highlights the ways in which CHWs are embedded in the community, well accepted by caregivers, and preferred agents for delivering health interventions [43]. Supporting the wider efforts to strengthen community health systems while integrating new nurturing care elements into the WBOT scope of work may be a way to promote child health and development in the future, with more evidence and insights of the effectiveness of specific interventions.

While there were some evident challenges to adoption among both CHWs and caregivers, the reported phenomenon of eventually adopting and accepting the intervention after initial hesitation is an important finding for ensuring successful recruitment and adoption in future interventions. It further points to the importance of broader and earlier community and stakeholder engagement, comprehensive and clear information regarding the intervention, and the need for better understanding the determinants of participation in research or interventions in this setting [44]. Nevertheless, the positive views expressed by caregivers echo other research on nurturing care interventions in LMICs [4], as caregivers tend to value the focus on children's wellbeing and learning in such interventions. Such findings are promising in terms of ensuring demand for the intervention if implemented more widely.

There are other practical lessons to be drawn from this study for future implementation and evaluation of Amagugu Asakhula or other interventions targeting early childhood health and development. For example, the fidelity findings indicate that there is a need for more thorough, accessible, and effective training of CHWs when it comes to both the intervention content and its delivery. Inaccurate reporting had a strong impact on data quality, demonstrating the need for enhanced training. This issue is especially pertinent when it comes to any expected research contributions, such as recruitment of eligible participants, carrying out the consent process with caregivers, and collecting basic questionnaire data or any other data CHWs are responsible for collecting. In particular, these concerns will need to be resolved if a definitive trial is carried out to ensure quality of outcome data, and that ethical standards are adhered to in 
obtaining caregivers' informed and voluntary consent for participation. In addition to more comprehensive training, the findings suggest a need to develop specific strategies to monitor delivery and ensure the fidelity of the intervention in order to accurately evaluate the effectiveness of the intervention in the future [45]. One approach is to allow for greater flexibility in future evaluations [10], with the aim to use process evaluation methodology to examine how modifications influence delivery and outcomes.

This study raises questions about how to proceed based on findings from formative research, as no quantitative threshold or pre-specified progression criteria were set to determine whether to proceed to an effectiveness trial of the intervention. This is an often overlooked topic, and there are calls for increased transparency and guidance on this aspect of formative studies [11]. The qualitative methods employed, in combination with the assessment of multiple process evaluation concepts and a theoretical framework to guide this investigation, enabled multifaceted learning and a nuanced picture of the complexities of intervention delivery. While there were promising findings relating to acceptability and appropriateness, there were several concerning features in the data relating to feasibility, fidelity, and context that fundamentally speak against this particular delivery approach. Moreover, the findings support the optimisation of the intervention in the future, demonstrating that the chosen data collection and analysis methods were appropriate for fulfilling the aims of this study.

As SK was responsible for carrying out the entire study, it is important to consider the role of observer bias, which is a typical limitation of intervention and implementation research $[46,47]$. Such observer or research bias is likely to result in the exaggeration of positive aspects at the cost of more critical observations. Moreover, as the data collection took place at the PHC facility, participants may have felt uncomfortable expressing critical views despite efforts to ensure privacy. This is particularly important to consider given that SK (a White European woman) was an obvious cultural outsider in the context of the research, making it potentially very challenging to build trust and rapport in a meaningful way [48, 49]. Numerous site visits offered ample opportunities for informal discussions and observation of the functioning of the WBOT programme more broadly, and the sharing of critical opinions in interviews and FGDs also suggests that participants were not limiting their responses solely to positive views. Nevertheless, the results described here can be characterised as a research team with a predominantly foreign pose, writing for a predominantly foreign academic gaze [50], thus lacking the full nuances that a truly local perspective could offer.

A specific limitation of this study was the weak reliability and quality of data collected by the CHWs. As this shortcoming was identified through other data sources, it can be interpreted as triangulation providing a level of protection from falsified data. In addition to being somewhat challenged by the intrinsic limitations detailed above, external factors such as the strikes also hindered the optimal delivery of the Amagugu Asakhula intervention. Nonetheless, this study provides many important insights to support future research and implementation.

\section{Conclusions}

This feasibility study contributes to the optimisation and further testing of the Amagugu Asakhula intervention and can inform the development and implementation of other nurturing care interventions. Delivering the Amagugu Asakhula intervention through CHWs linked to a PHC facility was found to be acceptable to caregivers of preschool children. However, this delivery mode was not found to be feasible for further implementation and testing due to organisational constraints related to the WBOT system in South Africa. Delivery through CHWs who are linked to a community-based organisation is recommended while recognising challenges linked to scalability and sustainability. The effects of Amagugu Asakhula on child health and developmental outcomes should be evaluated through definitive trials accompanied by process evaluation.

\section{Supplementary Information}

The online version contains supplementary material available at https://doi. org/10.1186/s40814-021-00802-6.

Additional file 1:. Data collection instruments

\section{Abbreviations}

CHW: Community health worker; FGD: Focus group discussion; LMIC: Lowand middle-income countries; PHC: Primary health care; WBOT: Ward-based outreach team

\section{Acknowledgements}

We would like to thank all participating CHWs, caregivers, and facility representatives in Soweto for their contributions to the Amagugu Asakhula intervention and our research. We are grateful to our main contact at the primary health care facility who enabled access to the research site and provided guidance throughout the study. We thank the Johannesburg Health District for granting us permission to conduct this research at the facility in Soweto. We also want to thank Edna Bosire and Sasha Jugdav for note-taking assistance during the focus group discussions.

\section{Authors' contributions}

SK designed the study, carried out the analysis, and drafted the manuscript with input and supervision from all other authors. SK and CED obtained ethical approval and research permissions. SK and CED coordinated the study, and SK carried out all training, CHW recruitment, data collection, and data management. All authors read and approved the final manuscript.

\section{Funding}

Funding from the Cambridge MRC Doctoral Training Programme, the Oppenheimer Memorial Trust in South Africa, the Research Councils UK Newton Fund to the University of Cambridge (ES/N013891/1) and from the National Research Foundation to the University of the Witwatersrand (UID:98561) is gratefully acknowledged. The funders had no role in designing the study, data collection, analysis, interpretation, or writing the manuscript. 


\section{Availability of data and materials}

The datasets generated and/or analysed during the current study are not publicly available due to participants not being asked for permission for making the data publicly available. Anonymous excerpts from transcripts are available from the corresponding author on reasonable request.

\section{Ethics approval and consent to participate}

Ethical approval was obtained from the University of the Witwatersrand's Medical Human Research Ethics Committee (reference number: M181063). Permission to carry out the study was also obtained from the Johannesburg Health District Research Committee (DRC Ref. 2019-01-013). All participants gave informed written consent for participation, and for being audiorecorded if relevant.

\section{Consent for publication}

Not applicable.

\section{Competing interests}

The authors declare no competing interests

\section{Author details}

${ }^{1}$ MRC Epidemiology Unit, UKCRC Centre for Diet and Activity Research (CEDA R), University of Cambridge, Cambridge, UK. ${ }^{2}$ SAMRC/Wits Developmental Pathways for Health Research Unit (DPHRU), School of Clinical Medicine, Faculty of Health Sciences, University of the Witwatersrand, Johannesburg, South Africa. ${ }^{3}$ Faculty of Medicine and Health Sciences, School of Health Sciences, University of East Anglia, Norwich, UK. ${ }^{4}$ Division of Exercise Science and Sports Medicine, Department of Human Biology, Faculty of Health Sciences, University of Cape Town, Cape Town, South Africa.

\section{Received: 29 July 2020 Accepted: 16 February 2021}

\section{Published online: 27 February 2021}

\section{References}

1. Britto PR, Lye SJ, Proulx K, Yousafzai AK, Matthews SG, Vaivada T, et al. Nurturing care: promoting early childhood development. Lancet. 2017; 389(10064):91-102.

2. Black MM, Walker SP, Fernald LCH, Andersen CT, DiGirolamo AM, Lu C, et al. Early childhood development coming of age: science through the life course. Lancet. 2017;389(10064):77-90.

3. Draper CE, Howard SJ, Rochat TJ. Feasibility and acceptability of a homebased intervention to promote nurturing interactions and healthy behaviours in early childhood: the Amagugu Asakhula pilot study. Child Care Health Dev. 2019:45(6):823-31.

4. Britto PR, Singh M, Dua T, Kaur R, Yousafzai AK. What implementation evidence matters: scaling-up nurturing interventions that promote early childhood development. Ann N Y Acad Sci. 2018;1419(1):5-16.

5. Tomlinson M, Hunt X, Rotheram-Borus MJ. Diffusing and scaling evidencebased interventions: eight lessons for early child development from the implementation of perinatal home visiting in South Africa. Ann N Y Acad Sci. 2018;1419(1):218-29.

6. Yousafzai AK, Aboud F. Review of implementation processes for integrated nutrition and psychosocial stimulation interventions. Ann N Y Acad Sci. 2014;1308(1):33-45.

7. Moore GF, Audrey S, Barker M, Bond L, Bonell C, Hardeman W, et al. Process evaluation of complex interventions: Medical Research Council guidance. BMJ. 2015:350:h1258.

8. Craig P, Dieppe P, Macintyre S, Michie S, Nazareth I, Petticrew M. Developing and evaluating complex interventions: the new Medical Research Council guidance. BMJ. 2008;337.

9. Bowen DJ, Kreuter M, Spring B, Cofta-Woerpel L, Linnan L, Weiner D, et al. How we design feasibility studies. Am J Prev Med. 2009;36(5):452-7.

10. O'Cathain A, Croot L, Duncan E, Rousseau N, Sworn K, Turner KM, et al. Guidance on how to develop complex interventions to improve health and healthcare. BMJ Open. 2019;9(8):e029954.

11. Hallingberg $B$, Turley $R$, Segrott J, Wight $D$, Craig P, Moore $L$, et al. Exploratory studies to decide whether and how to proceed with full-scale evaluations of public health interventions: a systematic review of guidance. Pilot Feasibility Stud. 2018 Dec;4(1).

12. O'Cathain A, Hoddinott P, Lewin S, Thomas KJ, Young B, Adamson J, et al. Maximising the impact of qualitative research in feasibility studies for randomised controlled trials: Guidance for researchers. Pilot Feasibility Stud. 2015;1(1):32.

13. Klingberg S, Draper CE, Micklesfield LK, Benjamin-Neelon SE, van Sluijs EMF. Childhood obesity prevention in Africa: a systematic review of intervention effectiveness and implementation. Int J Environ Res Public Health. 2019; 16(7):1212.

14. Limbani F, Goudge J, Joshi R, Maar MA, Jaime Miranda J, Oldenburg B, et al. Process evaluation in the field: global learnings from seven implementation research hypertension projects in low-and middle-income countries. BMC Public Health. 2019;19(1):953.

15. Rochat TJ, Mkwanazi N, Bland R. Maternal HIV disclosure to HIV-uninfected children in rural South Africa: a pilot study of a family-based intervention. BMC Public Health. 2013:13(1).

16. Rochat TJ, Mitchell J, Stein A, Mkwanazi NB, Bland RM. The Amagugu intervention: a conceptual framework for increasing HIV disclosure and parent-led communication about health among HIV-infected parents with HIV-uninfected primary school-aged children. Front Public Health. 2016:31:4.

17. Rochat TJ, Stein A, Cortina-Borja M, Tanser F, Bland RM. The Amagugu intervention for disclosure of maternal HIV to uninfected primary schoolaged children in South Africa: a randomised controlled trial. Lancet HIV. 2017:4(12):e566-76.

18. World Health Organization. Primary health care systems (PRIMASYS): case study from South Africa, abridged version. Geneva; 2017.

19. Mhlongo EM, Lutge E. The roles, responsibilities and perceptions of community health workers and ward-based primary health care outreach teams (WBPHCOTs) in South Africa: a scoping review protocol. Syst Rev. 2019 Dec;8(1)

20. Nyalunga S, Ndimande J, Ogunbanjo G, Masango-Makgobela A, Bogongo T. Perceptions of community health workers on their training, teamwork and practice: a cross-sectional study in Tshwane district, Gauteng. South Africa. South African Fam Pract. 2019;61(4):144-9.

21. Kinkel HF, Marcus T, Memon S, Bam N, Hugo J. Community oriented primary care in Tshwane District, South Africa: assessing the first phase of implementation. African J Prim Health Care Fam Med. 2013:5(1):36-44.

22. Le Roux KWDP, Couper I. Rural district hospitals - essential cogs in the district health system - and primary healthcare re-engineering. South African Med J. 2015;105(6).

23. O'Brien BC, Harris IB, Beckman TJ, Reed DA, Cook DA. Standards for reporting qualitative research. Acad Med. 2014;89(9):1245-51.

24. Hoffmann TC, Glasziou PP, Boutron I, Milne R, Perera R, Moher D, et al. Better reporting of interventions: template for intervention description and replication (TIDieR) checklist and guide. BMJ. 2014;7:348.

25. Yousafzai AK, Aboud FE, Nores M, Kaur R. Reporting guidelines for implementation research on nurturing care interventions designed to promote early childhood development. Ann N Y Acad Sci. 2018;1419(1):2637

26. Vorster $\mathrm{H}$, Badham J, Venter $\mathrm{C}$. An introduction to the revised food-based dietary guidelines for South Africa. South African J Clin Nutr. 2013;26(3):S1164.

27. Draper CE, Tomaz SA, Biersteker L, Cook CJ, Couper J, de Milander M, et al. The South African 24-hour movement guidelines for birth to 5 years: an integration of physical activity, sitting behavior, screen time, and sleep. J Phys Act Health. 2020;17(1):109-19.

28. Vorster H, Badham J, Venter C. An introduction to the revised food-based dietary guidelines for South Africa. S Afr J Clin Nutr. 2013;26:S1-S164.

29. Bourne LT. South African paediatric food-based dietary guidelines. Matern Child Nutr. 2007:3(4):227-9.

30. Peters DH, Adam T, Alonge O, Agyepong IA, Tran N. Republished research: implementation research: what it is and how to do it. Br J Sports Med. 2014; 48(8):731-6.

31. Braun V, Clarke V. Using thematic analysis in psychology. Qual Res Psychol. 2006:3(2):77-101.

32. Braun V, Clarke V. Reflecting on reflexive thematic analysis. Qual Res Sport Exerc Health. 2019:11(4):589-97.

33. Braun V, Clarke V. One size fits all? What counts as quality practice in (reflexive) thematic analysis? Qual Res Psychol. 2020;

34. Smith B, McGannon KR. Developing rigor in qualitative research: problems and opportunities within sport and exercise psychology. Int Rev Sport Exerc Psychol. 2018;11(1):101-21.

35. Smith S, Deveridge A, Berman J, Negin J, Mwambene N, Chingaipe E, et al. Task-shifting and prioritization: a situational analysis examining the role and 
experiences of community health workers in Malawi. Hum Resour Health. 2014;2:12(1).

36. Kok MC, Dieleman M, Taegtmeyer M, Broerse JEW, Kane SS, Ormel H, et al. Which intervention design factors influence performance of community health workers in low- and middle-income countries? A systematic review. Vol. 30, Health Policy and Planning. Oxford University Press; 2015. p. 120727.

37. John A, Newton-Lewis T, Srinivasan S. Means, Motives and opportunity: determinants of community health worker performance. BMJ Glob Health. 2019;1:4(5).

38. Yousafzai AK, Rasheed MA, Siyal S. Integration of parenting and nutrition interventions in a community health program in Pakistan: an implementation evaluation. Ann N Y Acad Sci. 2018;1419(1):160-78.

39. Ballard M, Montgomery P. Systematic review of interventions for improving the performance of community health workers in low-income and middleincome countries. BMJ Open. 2017;7(10):e014216.

40. Austin-Evelyn K, Rabkin M, Macheka T, Mutiti A, Mwansa-Kambafwile J, Dlamini T, et al. Community health worker perspectives on a new primary health care initiative in the Eastern Cape of South Africa. PLoS One. 2017; 12(3):e0173863.

41. Zambruni JP, Rasanathan K, Hipgrave D, Miller NP, Momanyi M, Pearson L, et al. Community health systems: allowing community health workers to emerge from the shadows. Lancet Glob Health. 2017;5(9):e866-7.

42. Cometto G, Ford N, Pfaffman-Zambruni J, Akl EA, Lehmann U, McPake B et al. Health policy and system support to optimise community health worker programmes: an abridged WHO guideline. Lancet Glob Health. 2018; 6(12):e1397-404

43. Draper CE, Bosire E, Prioreschi A, Ware L, Cohen E, Lye SJ, et al. Urban young women's preferences for intervention strategies to promote physical and mental health preconception: a Healthy Life Trajectories Initiative (HeLTI). Prev Med Reports. 2019;1:14.

44. Draper C, Ware L, Prioreschi A, Lye S, Norris S. "Honestly speaking, it's the..." money: pilot implementation of a preconception health trial in South Africa. (forthcoming, in review). SAGE Open Med. 2020

45. Bragstad LK, Bronken BA, Sveen U, Hjelle EG, Kitzmüller G, Martinsen R, et al. Implementation fidelity in a complex intervention promoting psychosocial well-being following stroke: an explanatory sequential mixed methods study. BMC Med Res Methodol. 2019;19(1):59.

46. de Villiers A, Steyn NP, Draper CE, Hill J, Dalais L, Fourie J, et al. Implementation of the HealthKick intervention in primary schools in lowincome settings in the Western Cape Province, South Africa: a process evaluation. BMC Public Health. 2015;15:818.

47. Angrosino MV. Observer Bias. In: The SAeGE Encyclopedia of Social Science Research Methods. SAGE Publications; 2004. p. 758-9.

48. Kalinga C. Caught between a rock and a hard place: navigating global research partnerships in the global South as an indigenous researcher. J African Cult Stud. 2019;31(3):270-2.

49. Liamputtong P. Performing qualitative cross-cultural research. Cambridge: Cambridge University Press; 2010.

50. Abimbola S. The foreign gaze: authorship in academic global health. BMJ Glob Health. 2019;4(5):e002068.

\section{Publisher's Note}

Springer Nature remains neutral with regard to jurisdictional claims in published maps and institutional affiliations.

Ready to submit your research? Choose BMC and benefit from:

- fast, convenient online submission

- thorough peer review by experienced researchers in your field

- rapid publication on acceptance

- support for research data, including large and complex data types

- gold Open Access which fosters wider collaboration and increased citations

- maximum visibility for your research: over $100 \mathrm{M}$ website views per year

At BMC, research is always in progress.

Learn more biomedcentral.com/submissions 